\title{
Enhancing TREATs Pulsing Capabilities Using TREAT Upgrade Fuel
}

\author{
Kayla Beth Clements
}

August 2019

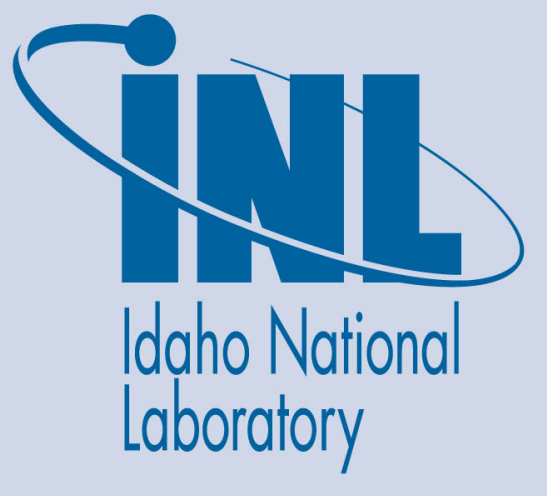

The INL is a U.S. Department of Energy National Laboratory operated by Battelle Energy Alliance 


\title{
Enhancing TREATs Pulsing Capabilities Using TREAT Upgrade Fuel
}

\author{
Kayla Beth Clements
}

\author{
August 2019 \\ Idaho National Laboratory \\ Idaho Falls, Idaho 83415 \\ http://www.inl.gov \\ Prepared for the \\ U.S. Department of Energy \\ Under DOE Idaho Operations Office \\ Contract DE-AC07-05ID14517
}




\section{WL

\section{Abstract}

The Transient Reactor Test Facility (TREAT) at Idaho National Laboratory is an air-cooled, thermal, heterogeneous facility used to test reactor materials in simulated accident conditions by inducing fission heating with intense neutron pulses ${ }^{1}$. TREAT operated from 1959 until 1994 with the primary goal of testing fast reactor fuels ${ }^{2}$ and was brought back online in 2017 to re-establish DOE's nuclear fuels transient testing capabilities ${ }^{3}$. Beginning in the late 1970s, Idaho National Laboratory worked in conjunction with other organizations to increase TREAT's capability for in-pile testing ${ }^{1}$. While these upgrades had not been implemented by the time the reactor was put into standby mode, new assemblies and graphite-urania fuel blocks with increased uranium concentrations had already been designed and fabricated. This project, as part of Idaho National Laboratory's Department of Reactor Physics, modeled the TREAT Upgrade (TU) fuel assemblies in MCNP and implemented them in an existing TREAT mode. Calculations were performed using INL's high performance computer to find a critical combination of TU and standard fuel and this geometry's excess reactivity. Future work will include calculating a power coupling factor in the experiment, determining power peaking factors throughout the core, and assessing how much reactivity would be added by replacing the Inconel-625 cladding with silicon carbide.

\section{Modeling TREAT Upgrade Fuel}

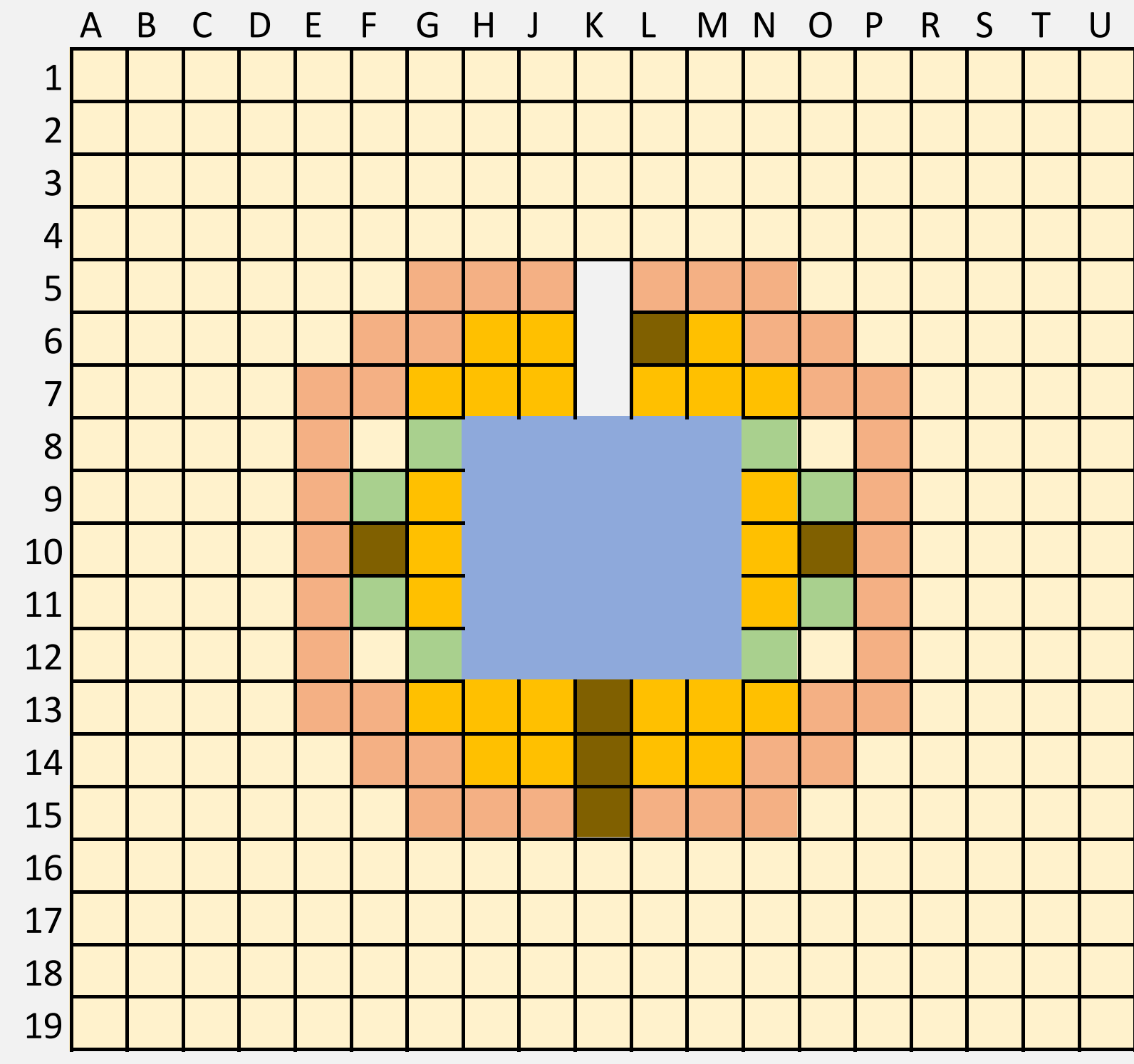

The figure on the right represents the concentration gradient of TU fuel, with green at the lowest uranium concentration of 1:5300 and red at the highest of 1:500. Buffer assemblies have the lowest concentrations of the TU assemblies, indicated by the green and yellow outer ring in the right image, and converter assemblies have the highest, indicated by the red and orange inner ring.

Left is a map of the intended Treat Upgrade core Fueled buffers, instrumented fuel, full and $4 \times 33 / 4$ converter fuel are different assembly types created for the TREAT Upgrade geometry. layout, where "driver fuel" is standard TREAT fuel.

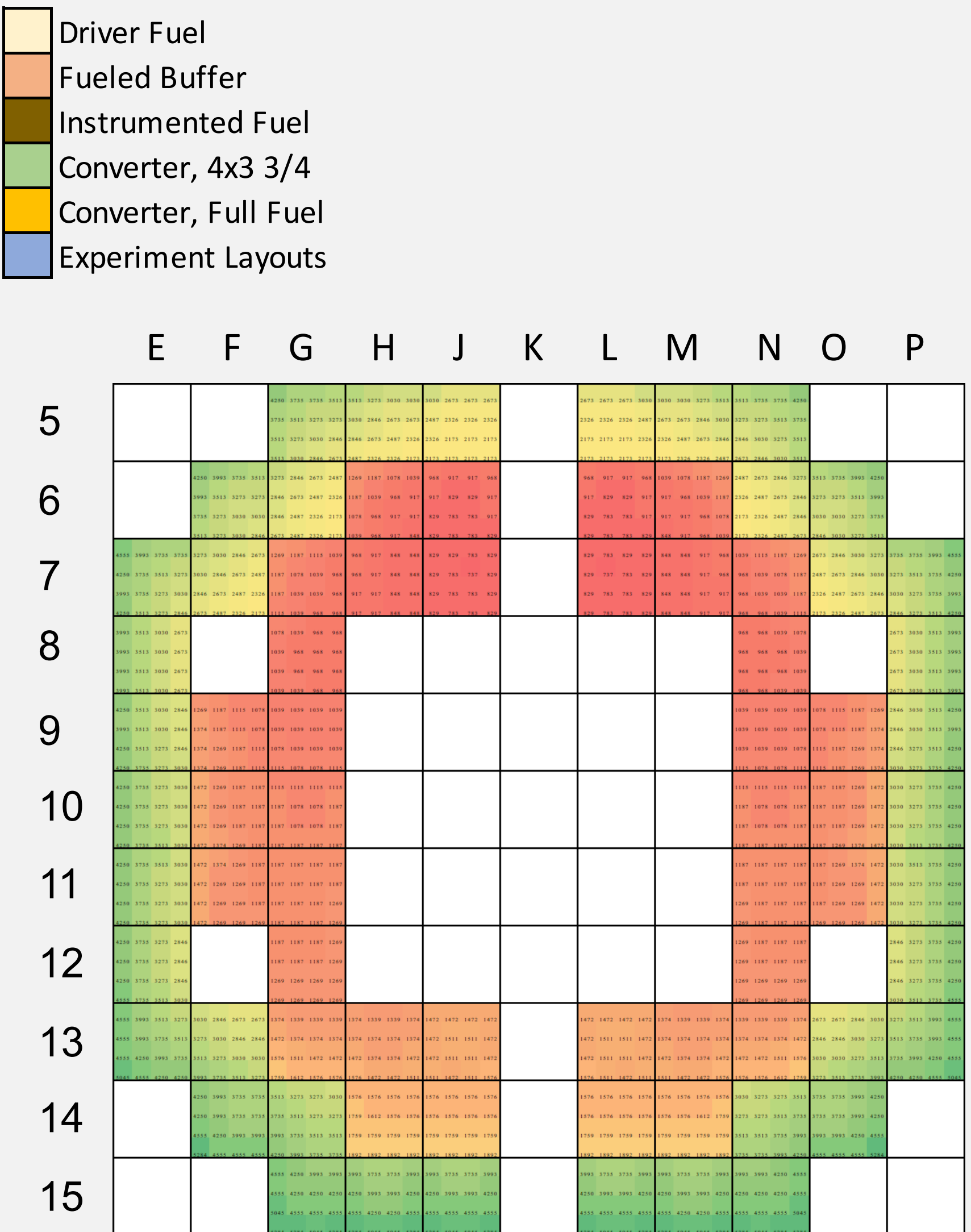

\section{Control Rod Movement}

Criticality is also controlled and maintained using control rods, which use boron carbide powder to absorb neutrons and can be inserted and removed into the reactor to control reactivity. Criticality with this arrangement was found to occur at a control rod insertion of approximately $66.3 \mathrm{~cm}$, where $0 \mathrm{~cm}$ is fully inserted and 147.32 is fully removed. The graph below shows the progression of $k_{\text {eff }}$ as the rod is removed.
1.08
eff was first calculated using MCNP by increasing the control rod position in increments of $10 \mathrm{~cm}$ until fully removed. One a window in which 60 and $70 \mathrm{~cm}$, the control
position was increased in position was increased in
increments of 1 , shown below.
0.98
0.96

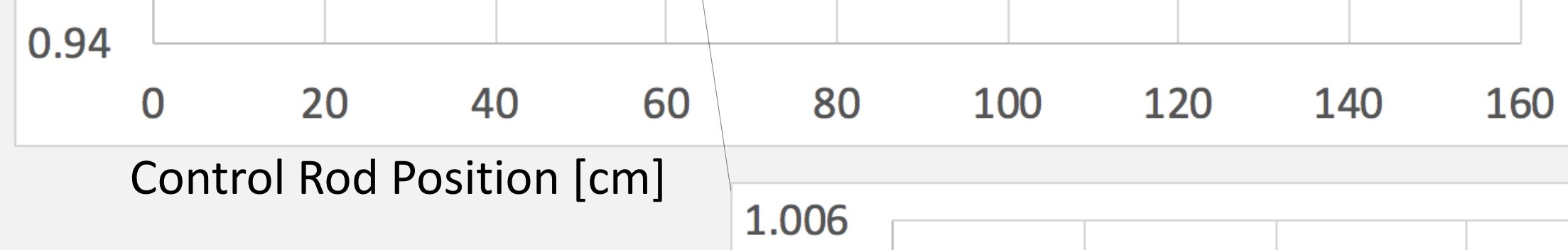
$\mathrm{K}_{\mathrm{eff}}$ was found to equal one $\quad 1.004$
at $66.3 \mathrm{~cm}$

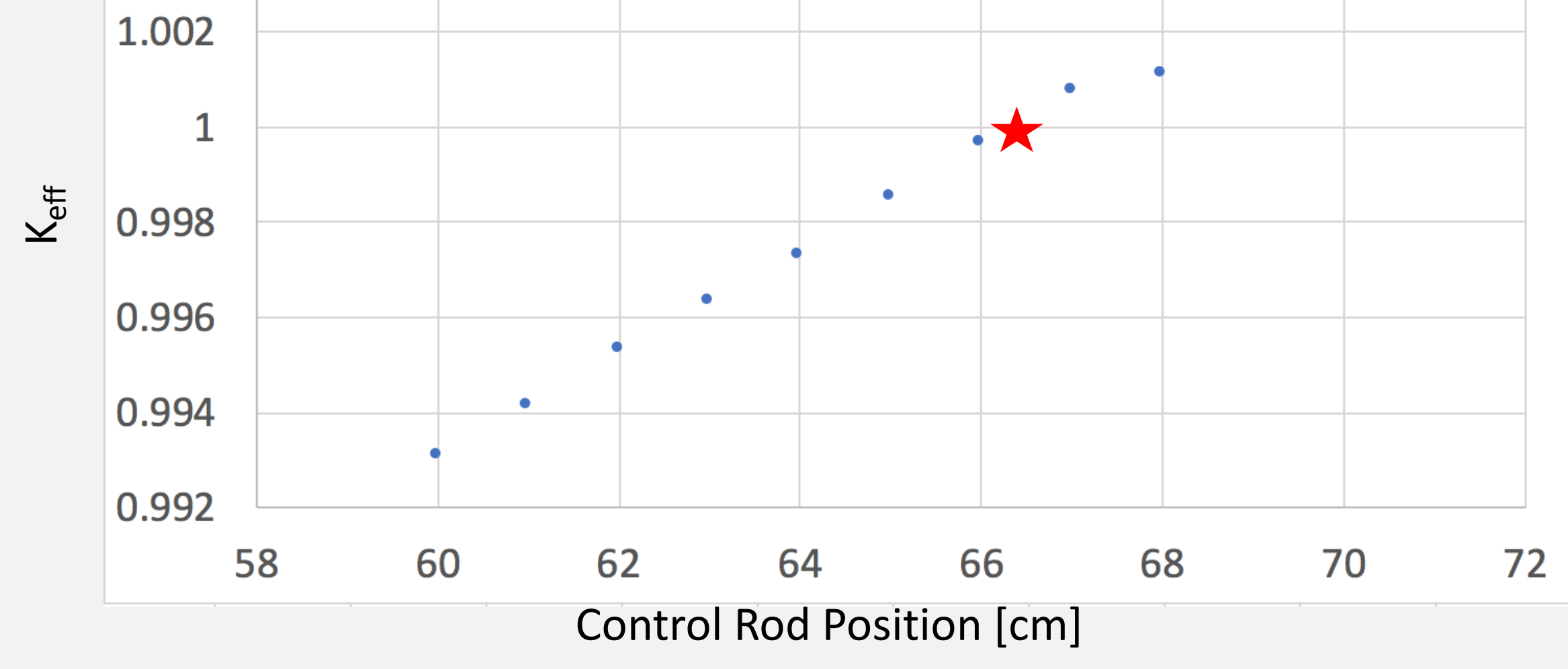

喜 1.02

\section{Acknowledgements}

I would like to thank my mentor, John D. Bess, for his support, guidance, and knowledge. I would also like to thank Nicolas E. Woolstenhulme and Andrew S. Chipman for their contributions. This was supported in part by the U.S. Department of Energy, Office of Science, Office of Workforce Development for Teachers and Scientists (WDTS) under the Science Undergraduate Laboratory Internships Program (SULI and made use of the resources of the High Performance Computing Center at INL, which is supported by the Office of Nuclear Energy of the U.S. Department of Energy and the Nuclear Science User Facilities under Contract No. DE-AC07-05ID14517.

\section{Standard Fuel vs. TREAT Upgrade Fuel}

The main differences between the Standard Fuel (SF) and TREAT Upgrade (TU) fuel from a modeling and neutronics perspective are as follows:

- Changes in construction materials

- Increase fuel length by $1^{\prime}$ and resulting decrease in reflector length

- Continuous outer cladding

- Increase in uranium concentration in fuel and introduction of azimuthal C/U ratio variation

SF vs. TU Materials and Dimensions

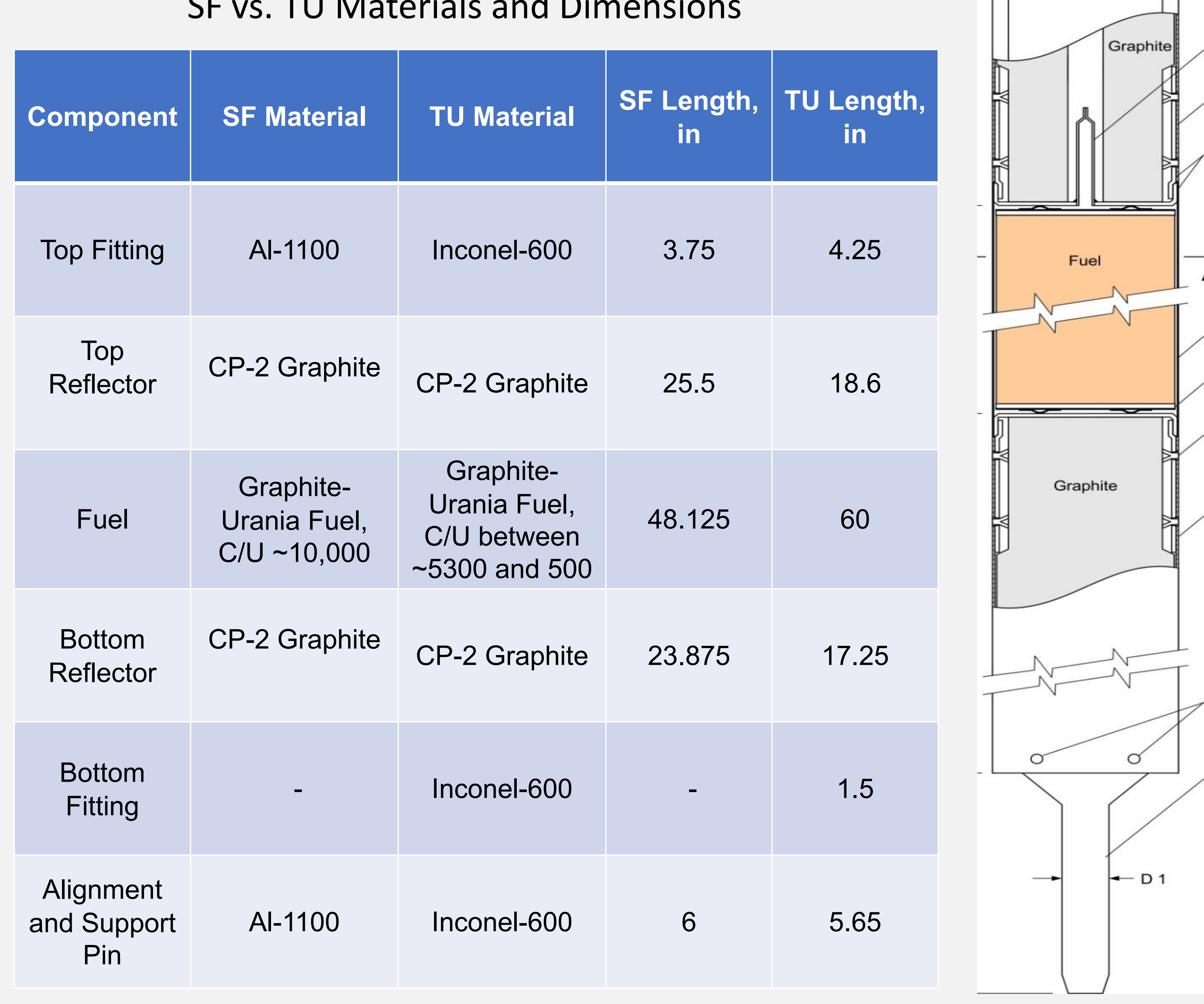

\section{Achieving Criticality}

Various combinations of TU and standard fuel were used to find a critical geometry. Standard fuel has a $\mathrm{C} / \mathrm{U}$ ratio of 10,000 , so the buffer $\mathrm{TU}$ fuel with the lowest uranium concentrations were used first. Criticality was found to occur using assemblies $\mathrm{F} 7,07$, G6, N6, F13, and 013 from the intended TU arrangement, placed at the six fuel locations closest to the experiment as shown right.
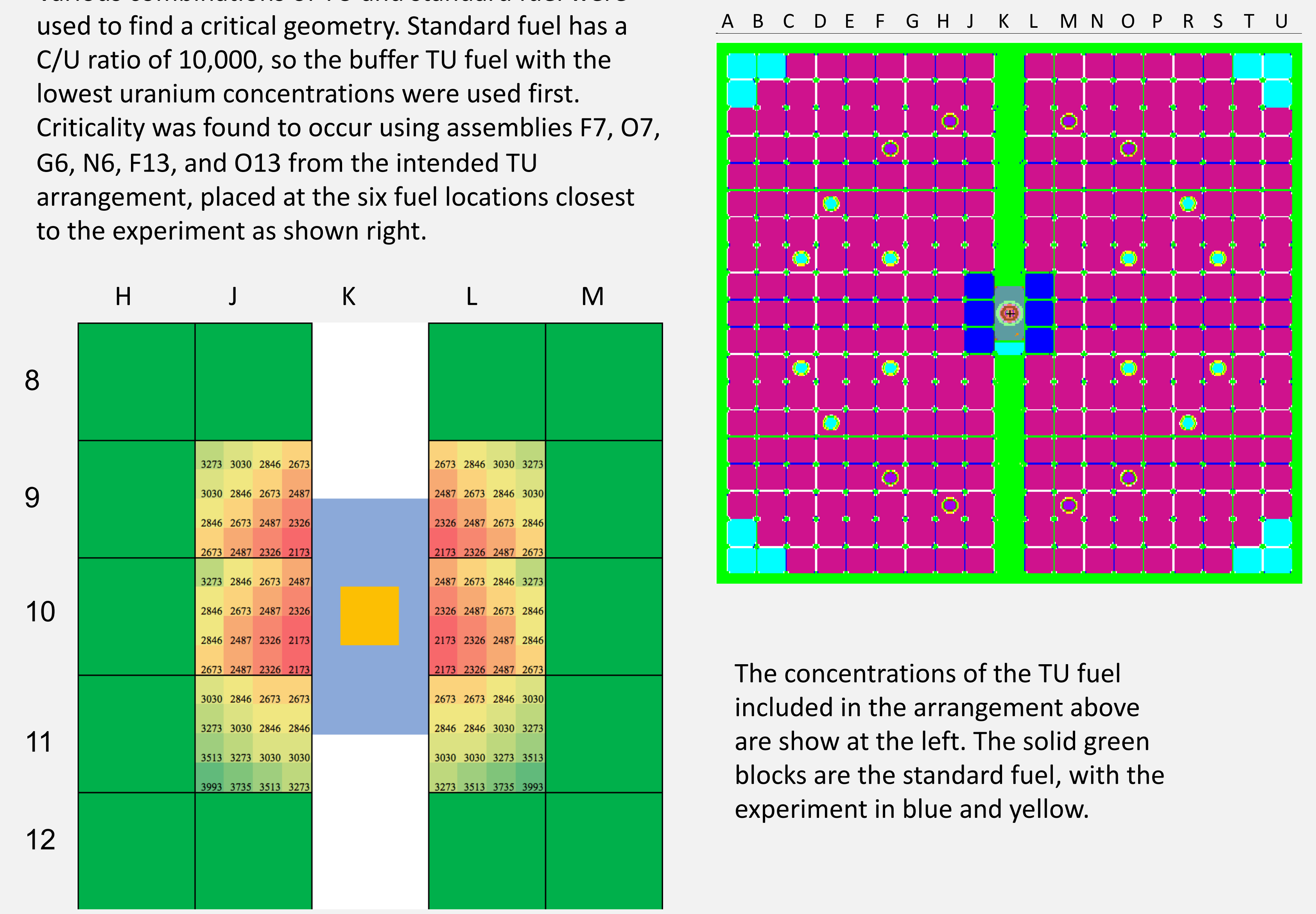

The concentrations of the TU fuel included in the arrangement above are show at the left. The solid green blocks are the standard fuel, with the experiment in blue and yellow.

\section{Future Work}

\section{- Calculate a power coupling factor}

- Ratio of energy in test fuel to energy dumped into core

Goal value between 0.7 and 1.2, to confirm that too much or too little energy isn't being put into the fuel relative to the entire core

- Calculate power peaking factors

- Ratio of assembly's energy deposition to core average energy deposition

Used to confirm that no one assembly in the core is undergoing much higher thermal stress than the rest of the core and to determine which assembly is the limiting assembly

- Assess added reactivity from switching Inconel-625 cladding to silicon carbide

- The nickel in Inconel causes it to absorb many more neutrons than silicon carbide, so switching to silicon carbide would boost excess reactivity in the core.

\section{References}

1. M. T. Kalman, M. A. Caruso, D. A. Prelewicz, G. B. Peeler,H. J. E. Jr., M. K. Prabjakara, and B. P. Singh, "Interim design report for the treat upgrade full fuel assembly," Tech. Rep. (NUS Corporation, 1981).

2. "Treat fact sheet," Available at https://www.energy.gov/sites/prod/files/TREATI\%20Factl\%20Sheet.pdf. 3. "Doe national laboratory resumes operation of us transient test reactor," Available at

https://www.energy.gov/articles/doe-national-laboratory-resumes-operation-us-transient-test-reactor 